\title{
Aspectos controversos do conceito de necessidades de saúde e seus reflexos sobre a acessibilidade de medicamentos e serviços de saúde
}

\author{
Controversial aspects of the concept of health needs and their \\ impact on the accessibility of medicine and health services
}

Lia Hasenclever (https://orcid.org/0000-0003-1384-6323) ${ }^{1}$

Caroline Miranda (https://orcid.org/0000-0001-7126-2348) ${ }^{2}$

Gabriela Chaves (https://orcid.org/0000-0002-8347-6164) ${ }^{3}$

André Luís Almeida Peixoto (https://orcid.org/0000-0003-4349-2108) ${ }^{4}$

Leonardo Vidal Mattos (https://orcid.org/0000-0003-4800-0010) ${ }^{5}$

Juliana de Souza Viana (https://orcid.org/0000-0003-3358-4762) ${ }^{6}$
${ }^{1}$ Universidade Cândido Mendes. Rua Anita Peçanha 100, Parque São Caetano. 28040-320 Campos dos Goytacazes RJ Brasil. lia@ie.ufrj.br

${ }^{2}$ Programa de PósGraduação em Políticas Públicas, Estratégias e Desenvolvimento, Instituto de Economia, Universidade Federal do Rio de Janeiro. Rio de Janeiro RJ Brasil. ${ }^{3}$ Departamento de Política de Medicamentos e Assistência Farmacêutica, Escola Nacional de Saúde Pública Sergio Arouca, Fundação Oswaldo Cruz. Rio de Janeiro RJ Brasil.

${ }^{4}$ Instituto Federal de

Educação, Ciência e Tecnologia Fluminense. Campos dos Goytacazes RJ Brasil.

${ }^{5}$ Grupo de Pesquisa e Documentação sobre o Empresariamento da Saúde, Instituto de Estudos em Saúde Coletiva, Universidade Federal do Rio de Janeiro. Rio de Janeiro RJ Brasil.

${ }^{6}$ Faculdade de Medicina de Campos. Campos dos Goytacazes RJ Brasil.

\begin{abstract}
This article aimed to present the problems related to accessibility of health services by re-reading four case studies which illustrate the different dimensions of the definition of health needs and their impact on the accessibility of medicine and health services. The theoretical model of Vargas-Peláez et al. ${ }^{1}$, which allows a better understanding of the complexity of the definition of health needs and its impact on the demand for medicines and services, was adopted to re-read the case studies on the prices of patented medicines, the models of public provision, hemodynamic services, and prescription of anxiolytics. The results may shed some light on the complexity of the challenges to achieve health rights in a peripheral capitalist society where the production and distribution of goods and services is organized from the perspective of the market, even though public policies seek to regulate them. In this sense, it seems essential that the State plays a major role in guaranteeing equality in the achievement of social rights, not only influencing the industrial sector in reducing the prices of medicines and improving their supply, but also guaranteeing access to medicine and health services.
\end{abstract}

Key words Health policy, Industrial policy, Pharmaceutical services, Health needs, Accessibility
Resumo O objetivo do artigo é mostrar os problemas relativos à acessibilidade de serviços de saúde relendo quatro estudos de caso que ilustram as diferentes dimensões da definição de necessidades de saúde e os seus reflexos sobre a acessibilidade dos medicamentos e dos serviços de saúde. O modelo teórico de Vargas-Peláez et al. ${ }^{1}$, que permite um melhor entendimento da complexidade da definição das necessidades de saúde e os seus reflexos sobre a demanda de medicamentos e de serviços, foi adotado para reler os estudos de caso sobre preços dos medicamentos patenteados, os modelos de provisão pública, os serviços de hemodinâmica e a prescrição de ansiolíticos. Os resultados permitem iluminar a complexidade dos desafios presentes para obtenção dos direitos de saúde numa sociedade capitalista periférica em que a produção e a distribuição dos bens e serviços são organizadas na perspectiva do mercado ainda que as políticas públicas busquem regulá-las. Neste sentido, parece imprescindivel que o Estado tenha um papel preponderante na garantia da equidade para o alcance dos direitos sociais, não só estimulando a indústria para redução dos preços dos medicamentos e melhoria de sua provisão, como garantindo acesso aos medicamentos e serviços de saúde.

Palavras-chave Politica de saúde, Política industrial, Assistência farmacêutica, Necessidades de saúde, Acessibilidade 


\section{Introdução}

Segundo Soares ${ }^{2}$ a acessibilidade é uma característica do sistema de saúde relacionada à sua capacidade de fornecer bens e serviços necessários em uma perspectiva coletiva que abranja a totalidade dos cidadãos. Neste sentido, poderia se perguntar se o complexo econômico-industrial da saúde (CEIS) é capaz de ofertar bens e serviços necessários e disponibilizá-los ao usuário de formas a garantir a universalidade do sistema de saúde como um direito, previsto na Constituição Federal brasileira de 1988. Entende-se o termo CEIS como o conjunto de bens necessários para a provisão do sistema de saúde que engloba agentes públicos e privados na produção e prestação de serviços ${ }^{3}$.

Vários esforços têm sido direcionados para a consecução desse objetivo, através de políticas de saúde: a criação do Sistema Único de Saúde (SUS), em 1990, responsável pela universalização da proteção à saúde; a Política Nacional de Medicamentos (PNM), em 1998, marco para garantia dos medicamentos essenciais; e a Política de Assistência Farmacêutica, em 2004, ampliando a abrangência do conceito de assistência farmacêutica no sentido da integralidade ${ }^{4}$. São exemplos de políticas industriais e de inovação, relacionadas ao CEIS, a Política Industrial, Tecnológica e de Comércio Exterior (PITCE), que visa induzir a competitividade da indústria brasileira e a inovação, a Política de Desenvolvimento Produtivo (PDP), com vistas a ampliar a competitividade e a inovação e a Política Brasil Maior (PMB), visando crescimento sustentável e inclusivo puxado pela inovação tecnológica ${ }^{5}$. Entretanto, trinta anos depois da promulgação da Constituição, poucos e avançou na construção de um sistema de saúde universal ${ }^{4,6}$.

O estudo da acessibilidade do sistema de saúde pelos cidadãos em uma perspectiva universal é um tema extremamente complexo, pois envolve interesses públicos e privados. A hipótese do artigo é que, parte dessa complexidade poderia ser melhor percebida com a análise do grande número de elementos intervenientes na definição de necessidades de saúde que acabará por refletir na acessibilidade do sistema. Ou seja, as diferentes visões acerca de necessidades de saúde de cada um dos sistemas, o primeiro na perspectiva da saúde, e o segundo na perspectiva do mercado, explicam em parte o problema do difícil alcance da acessibilidade.

Isso ocorre porque os sistemas de saúde são construções sociais, com dependências de traje- tórias específicas, estruturas de poder, interesses e interdependências permeadas por valores $e$ princípios dos stakeholders (governo, profissionais dos serviços de saúde, complexo médico-industrial, provedores de serviços entre outros). Os distintos interesses de cada stakeholder e dos objetivos de políticas, com perspectivas distintas na definição do conceito de necessidades de saúde, acaba impactando na acessibilidade do sistema de saúde.

Segundo Vargas-Peláez et al. ${ }^{1}$, o medicamento na perspectiva da saúde é encarado como um bem social, ou, como a literatura econômica o define, um bem público, que tem o propósito de prevenir e solucionar problemas de saúde de forma ilimitada e sem custo. Ainda segundo os mesmos autores, na perspectiva do mercado, o medicamento é uma mercadoria como outra qualquer e é produzido e vendido pelas empresas que buscam constantes inovações e sua comercialização através do marketing, dirigido, principalmente, aos profissionais médicos.

A acessibilidade dos sistemas de saúde irá depender, portanto, de uma série de dimensões da definição de necessidades de saúde, sempre influenciadas de forma não balanceada pelas perspectivas da saúde e do mercado. Ela é influenciada por aspectos normativos, tais como a definição da Organização Mundial da Saúde (OMS) quanto à essencialidade dos medicamentos e o direito à saúde, a garantia de acesso aos medicamentos e às políticas e regulações, a questão das patentes, a produção de genéricos e a produção pública e a judicialização. Está relacionada também a definição de direitos humanos, a valores de justiça social, equidade e eficiência. E, por fim, a demanda por saúde, articulada com o comportamento e a ação do usuário para obter serviços ou produtos de saúde, e a organização da produção e consumo de bens irão também refletir nas condições de acessibilidade do sistema de saúde ${ }^{7}$.

O grande desafio é como conciliar as diferentes perspectivas de forma a garantir um equilíbrio justo entre as forças da saúde e do mercado. No Brasil, a produção e a provisão de bens e serviços de saúde são realizadas, majoritariamente, pela iniciativa privada $^{8}$, apesar da existência do SUS e da produção pública dos laboratórios farmacêuticos oficiais (LFO). Logo, as dificuldades de se manter um equilíbrio entre a perspectiva da saúde e do mercado tornam-se ainda mais delicadas.

O objetivo do artigo é mostrar as contradições que se apresentam para a acessibilidade de serviços de saúde, por meio da releitura de qua- 
tro estudos de caso realizados previamente pelos autores à luz do modelo de Vargas-Peláez et al. ${ }^{1}$ para evidenciar as contradições entre as distintas dimensões de necessidades de saúde acima apresentadas e os desacordos entre os distintos stakeholders sobre as perspectivas de saúde, assim como a dificuldade em se obter uma visão equilibrada e justa entre essas perspectivas para a provisão de medicamentos e de serviços de saúde. Além de evidenciar as contradições das definições de necessidades de saúde, a releitura dos casos estudados ilustra também as dificuldades reais de se alcançar a universalidade e a integralidade da acessibilidade aos serviços de saúde e medicamentos, garantidos e cobertos pelo sistema de saúde público brasileiro.

O artigo está organizado em três seções, além dessa introdução. Na primeira seção, apresenta-se o quadro de análise e o método utilizado para releitura dos estudos de caso realizados na perspectiva das várias dimensões da definição de necessidades de saúde e como elas influenciam a acessibilidade de medicamentos e serviços de saúde. Na segunda seção, desenvolve-se a releitura dos quatro estudos de caso na perspectiva indicada. Finalmente, nas considerações destacamse as inúmeras dificuldades para o alcance de um melhor equilíbrio entre as forças antagônicas da saúde e do mercado, ilustradas pelos casos apresentados.

\section{Abordagem teórica e métodos}

O modelo teórico construído por Vargas-Peláez et al. ${ }^{1}$ foi adotado como base para a releitura dos estudos desenvolvidos por pesquisadores da Universidade Federal do Rio de Janeiro (UFRJ), da Universidade Cândido Mendes (UCAM) e da Escola Nacional de Saúde Pública Sergio Arouca (ENSP). As principais contribuições do modelo adotado são trazer a complexidade da definição das necessidades de saúde para a demanda de medicamentos e serviços de saúde e considerar os interesses antagônicos da sociedade - o lado do mercado e o lado da saúde - no entendimento dos problemas para o alcance de um sistema público de saúde universal.

Segundo os autores do modelo, pode-se observar a existência de várias abordagens diferentes sobre necessidades de saúde, principalmente pela sua complexidade e pela não uniformidade do conceito. Esses autores elegeram três dos principais estudos encontrados na literatura que abordam a problemática das definições de neces- sidades, são eles: Bradshaw ${ }^{9}$, Willard ${ }^{10}$ e Max-Neef et al. ${ }^{11}$.

Os estudos de Max-Neef et al. ${ }^{11}$ e Willard ${ }^{10}$ enfatizam a perspectiva histórica e social das necessidades de saúde, mostrando que esta é uma noção, um constructo, que varia com o tempo e que uma série de valores subjetivos e evolutivos relativos em como a sociedade se estrutura interferem nas funções, expectativas e demandas sociais e, consequentemente, geram visões distintas de necessidades de saúde.

O estudo de Bradshaw ${ }^{9}$ concorda com os dois primeiros autores, e afirma que o conceito de necessidade social, inclusive as médicas, é inerente à ideia de serviço social e a história dos serviços sociais é a história do reconhecimento das necessidades e da organização da sociedade para atendê-las. A partir desse pressuposto, o autor pragmaticamente propõe quatro dimensões da definição de necessidades de saúde.

A primeira delas é a dimensão normativa, que é aquela em que o especialista, ou profissional administrador ou cientista social, define como necessidade em qualquer situação. Assim, a definição normativa da necessidade, segundo esse autor, pode ser diferente de acordo com a orientação dos valores do especialista - em seus julgamentos sobre a quantidade de recursos que devem ser dedicados ao atendimento da necessidade ou se as habilidades disponíveis podem ou não resolver o problema. Os padrões normativos mudam com o tempo, tanto como resultado da evolução do conhecimento, como da mudança dos valores da sociedade sobre a definição dos medicamentos a serem cobertos pelo sistema de saúde.

A segunda dimensão da definição de necessidade diz respeito à necessidade sentida, em que necessidade se iguala a desejo/querer. Ao avaliar a necessidade de um serviço, a população é questionada se acha se precisa daquilo. Numa democracia, pode-se imaginar que a necessidade sentida seria um componente importante de qualquer definição de necessidade. A necessidade sentida é, por si só, uma medida inadequada da "necessidade real", sendo limitada pelas percepções do indivíduo a uma prescrição médica ou ao efeito do marketing farmacêutico.

A terceira dimensão da definição é a necessidade expressa ou demanda, ou seja, é a necessidade sentida transformada em ação. Sob esta definição, a necessidade total é definida como a necessidade das pessoas que demandam um serviço. Não se demanda um serviço, a menos que se sinta uma necessidade, mas, por outro lado, 
é comum que a necessidade expressa não seja atendida. A necessidade expressa e não atendida é comumente registrada nos serviços de saúde em listas de espera que são tomadas como uma medida da necessidade não atendida.

Por fim, a quarta dimensão da definição é a necessidade comparativa, que é uma medida de necessidade obtida pelo estudo das características da população na recepção de um serviço. Se houver pessoas com características semelhantes que não recebam um serviço, elas estarão necessitadas.

A partir desse entendimento do conceito de necessidades de saúde, também adotado por esse artigo, e de suas várias dimensões, Vargas-Peláez et al. ${ }^{1}$ concluem que quando essas várias dimensões da definição de necessidades de saúde não são alcançadas há uma barreira para acessibilidade do sistema de saúde. O modelo sugerido pelos autores destaca o fato de que em uma sociedade capitalista, que tem a produção e os serviços organizados pelo mercado e os bens e serviços são mercadorias, é preciso que o Estado garanta não só a cada indivíduo oportunidades para o exercício de sua condição de cidadania, como um sistema de saúde eficiente e igualitário que permita a acessibilidade dos medicamentos de acordo com a normativa de direitos humanos ${ }^{12}$.

Ao Estado caberá também estabelecer políticas públicas de estímulo e regulação do mercado privado, a exemplo das políticas de inovação e propriedade intelectual e de controle de preços e garantias sanitárias. Essas políticas têm a finalidade de promover o desenvolvimento de setores econômicos fundamentais para o aumento da eficiência econômica, geração de divisas estrangeiras, difusão de tecnologias e expansão dos níveis de emprego e renda ${ }^{13}$.

Estes são os aspectos considerados no modelo teórico selecionado para releitura dos estudos de caso. O procedimento metodológico deste artigo foi reler os resultados de quatro estudos de caso já realizados e publicados à luz do modelo teórico de Vargas-Peláez et al. ${ }^{1}$. Os estudos de caso focam em diferentes aspectos da acessibilidade de medicamentos e de serviços no SUS. A contribuição esperada é que a exposição das várias dimensões da definição de necessidades de saúde e das diferentes perspectivas dos stakeholders em relação à saúde e ao mercado, explicitadas pelo modelo, possam ajudar na interpretação e compreensão das dificuldades para a acessibilidade dos medicamentos e dos serviços de saúde apontadas nos estudos de caso. É importante deixar claro que o objetivo do artigo não é a realização dos estudos de caso, mas somente a releitura de seus resultados a partir do modelo teórico descrito anteriormente.

\section{Resultados e discussão dos estudos de casos}

Nesta seção apontam-se as dificuldades reais de acessibilidade aos medicamentos e aos serviços de saúde, no Brasil, estudados em quatro estudos de caso, desenvolvidos na UFRJ, na UCAM e na ENSP. Os dois primeiros estudos analisam a questão da acessibilidade pelo lado da oferta em duas dimensões: disponibilidade de medicamentos a preços justos e modelos de provisão de medicamentos para o sistema de saúde. Os dois outros estudos de caso analisam a questão da acessibilidade pelo lado da demanda de serviços de hemodinâmica, que são dependentes de equipamentos para a sua realização, e da prescrição e demanda de medicamentos ansiolíticos.

O primeiro estudo ${ }^{14}$ ilustra as necessidades normativas de uma perspectiva da disponibilidade de oferta de medicamentos a preços justos. Entre os fatores que explicam a definição de necessidade normativa estão em que medida a regulação patentária e a produção local (de medicamentos genéricos e públicos) são elementos normativos relevantes para explicar parte dos problemas de acessibilidade dos medicamentos. A regulação patentária ilustra uma norma internacional e a sua necessidade de adequação ao país para que ela não se torne uma barreira à obtenção de preços justos e a produção local ilustra a atuação do Estado no fomento à oferta de bens procurando compensar os efeitos deletérios das barreiras patentárias.

O segundo estudo ${ }^{15}$ exemplifica a necessidade comparativa de uma perspectiva da oferta, ou seja, a capacidade do sistema de saúde de responder equitativamente às necessidades das pessoas e visando a acessibilidade de medicamentos. A definição está interligada aos direitos humanos, a valores de justiça social, equidade e eficiência. Cada sistema de saúde define a cobertura populacional e as fontes de recursos alocadas para garantir a acessibilidade nos sistemas de saúde. $\mathrm{O}$ estudo focou nos modelos de provisão e financiamento de medicamentos disponíveis hoje e nas suas contradições em alcançar a universalidade e a integralidade da acessibilidade de medicamentos.

O terceiro estudo ${ }^{16}$ elucida a definição de necessidades expressas de uma perspectiva da demanda de serviços de saúde. A acessibilidade aos 
serviços de saúde (por exemplo, organização, distribuição geográfica e questões financeiras) e os fatores facilitadores (por exemplo, status socioeconômico, percepção sobre o sistema e o direito à saúde) influenciam a possibilidade de acesso a este tipo de serviço que é dependente de equipamentos e por isso exige investimentos iniciais elevados para ser ofertado. O foco do estudo foi compreender aspectos de distribuição geográfica e financiamento desse tipo de serviços médicos que dificultam a acessibilidade aos serviços de hemodinâmica.

Por fim, o quarto estudo ${ }^{17}$ esclarece a definição de necessidades sentidas pelos pacientes na perspectiva da demanda. Os fatores que concorrem para explicar a categoria de necessidade sentida apontam a visão de necessidades criadas pelo mercado por meio do marketing farmacêutico (marcas e difusão junto ao prescritor), mas também a crença de que o progresso científico traz sempre aumento do bem-estar e a nova forma de tratamento é sempre melhor do que a velha, ilustrando o fato conhecido na literatura como “medicalização da vida" que leva à percepção dos medicamentos como uma necessidade de saúde. O foco do estudo foi na influência desses fatores no consumo de ansiolíticos em uma comunidade rural de Campos dos Goytacazes, município localizado no estado do Rio de Janeiro.

As quatro subseções a seguir resumem a releitura dos objetivos e dos resultados de cada um desses estudos realizados anteriormente à luz das definições de necessidades propostas no modelo, visando explicitar as contradições reais que se apresentam para o alcance de um sistema de saúde que garanta acessibilidade gratuita e universal. Essa releitura permitirá avançar em maior compreensão dos desafios observados em cada estudo e contribui para a busca de suas soluções.

\section{Disponibilidade da oferta de medicamentos a preços justos}

A principal motivação do primeiro estudo ${ }^{14}$ foi mostrar que, sem produção local não seria possível fazer uma política de saúde adequada para garantir a oferta de medicamentos a preços justos. Isso porque o mercado farmacêutico é extremamente imperfeito e a concorrência é toda baseada em inovação ${ }^{18}$. A produção e a inovação locais são importantes para se contra-arrestar o poder de monopólio das empresas estabelecidas, em geral multinacionais, mesmo que seja produzindo medicamentos genéricos ou similares. A partir da segunda metade dos anos 1990, o
Brasil trocou o monopólio europeu e americano da produção de medicamentos pelo monopólio chinês e indiano. As iniciativas da China e da Índia ao longo dos anos 1990 baratearam muito, respectivamente a produção de intermediários químicos e medicamentos, mas no início da década de 2000 seus preços voltaram a aumentar ${ }^{19}$. Então, sem produção local, a disponibilidade de oferta de medicamentos fica reduzida e sujeita a preços abusivos e inviáveis para o orçamento público.

Uma consequência disto é que a provisão de bens para o sistema de saúde torna-se dependente da importação e da disponibilidade de divisas. Durante a vigência da Convenção da União de Paris havia a possibilidade de não se patentear em certas áreas tecnológicas para países que, apesar de signatários, considerassem a sua acumulação de conhecimento na área insuficiente para justificar a manutenção de um regime de propriedade intelectual.Todavia, com a assinatura do Acordo sobre os Aspectos dos Direitos de Propriedade Intelectual Relacionados ao Comércio (em inglês, TRIPS), em 1994, essa possibilidade deixou de existir e o uso da estratégia de cópias de medicamentos, ainda com patentes vigentes,para aprendizado e produção local teve que ser abandonada em prol de novas estratégias para enfrentar as barreiras patentárias.

O estudo ilustra a definição de necessidade normativa, apresentada no modelo, na medida em que foca na importância das políticas de regulação, incluída a questão das patentes, da produção de genéricos e da produção pública, para que a disponibilidade de oferta de medicamentos a preços justos seja garantida após a mudança da norma internacional - assinatura do TRIPS. O estudo enfatiza a importância da regulação patentária e da produção local, tanto por meio do incentivo à produção de genéricos quanto à produção pública, como relevantes para a acessibilidade dos medicamentos.

Três casos de enfrentamento do monopólio, viáveis segundo as normas do TRIPS, praticados no Brasil, foram eleitos: um caso em que a patente já havia sido concedida, e dois casos de potenciais monopólios, isto é, casos em que os pedidos de patentes estavam apenas depositados. O desfecho do enfrentamento em cada um dos casos foi distinto: no caso do Efavirenz o governo decidiu por licença compulsória; no caso do Tenofovir, subsídio ao exame; no caso do Atazanavir, licença voluntária. Esses três casos ilustram diferentes iniciativas de produção local no contexto de negociação de preços, seguidas de desenvolvimento 
industrial, mas como será mostrado, que tiveram efeitos diferenciados sobre a redução de preços.

O principal achado do estudo ${ }^{14}$ foi que, conforme os desfechos caminhavam da licença compulsória para o subsídio ao exame e para a licença voluntária, o efeito desejado sobre a redução de preços decrescia. Ou seja, o efeito maior sobre a redução de preços foi obtido com a licença compulsória. Esta forma de enfrentamento mostrouse a maior ameaça para a concorrência porque usa a salvaguarda da obrigatoriedade de cessão dos direitos patentários, contestando diretamente o poder de monopólio da empresa detentora da patente. Em segundo lugar, o subsídio ao exame que resultou em não concessão da patente, mas permitiu uma redução de preços bem menos significativa. E, por último, a licença voluntária, em que a redução dos preços foi irrisória.

$\mathrm{O}$ achado comum entre os três casos estudados no artigo $^{14}$ é que o caminho para o fomento à concorrência é essencial para a obtenção de sucesso na redução de preços, mas não é suficiente. A lição apreendida, quando a regulação de preços não esteve atrelada à produção e à inovação locais, foi que o efeito sobre a redução dos preços não foi tão significativo devido às características intrínsecas do mercado farmacêutico.

Os aspectos iluminados pelo modelo de Vargas-Peláez et al. ${ }^{1}$ permitiram mostrar que os diferentes fatores - normas internacionais e políticas nacionais - interferem na disponibilidade da oferta de medicamentos a preços justos. E que nem sempre as perspectivas da saúde e do mercado, assim como os interesses dos vários stakeholders encontram um ponto adequado para que as duas perspectivas e os diferentes interesses sejam atendidos sem geração de conflitos, afetando, por fim, a acessibilidade de medicamentos por parte do sistema de saúde.

De fato, fica claro que o Ministério da Saúde buscou ajustar sua política de fomento de produção e inovação locais desde 2008, com a publicação da PDP e a criação do instrumento de parcerias de desenvolvimento produtivo em 2009, buscando conciliar os interesses dos stakeholders produtores de medicamentos, através do oferecimento da garantia do mercado público por até dez anos. As parcerias são realizadas entre os LFO, receptores da tecnologia, e as empresas nacionais e estrangeiras, transferidoras da tecnologia. A conclusão é que, apesar de a estratégia brasileira para o enfrentamento da barreira patentária ter evoluído, ela ainda apresenta algumas fragilidades.

A principal fragilidade é a falta de compromisso do próprio governo no investimento necessário em pesquisa e desenvolvimento, incluindo treinamento das equipes, nos LFO que são os receptores da tecnologia ${ }^{20}$. Este fato está colocando em risco a capacidade de absorção da tecnologia transferida. Os riscos são de médio e longo prazo. No médio prazo está se colocando em risco a capacidade de os LFO não estarem prontos para produzir o medicamento após o fim da parceria de transferência de tecnologia. No longo prazo, é que, à medida que novos produtos apareçam no mercado e sejam demandados para os tratamentos, se tenha que adquirir novamente a tecnologia de empresas estrangeiras por não ter se avançado na capacitação tecnológica e mantido a dependência.

A releitura do estudo destaca, portanto, a importância do instrumento de parcerias para o desenvolvimento produtivo como forma alternativa de enfrentamento da barreira patentária, mas ao mesmo tempo aponta para a necessidade de um maior compromisso do governo em investimentos nos LFO, para que estes possam exercer a sua funcionalidade nas parcerias.

\section{Sistema de provisão de medicamentos}

A motivação do segundo estudo revisita$\mathrm{do}^{15}$ foi analisar como se financia e se organiza a provisão de medicamentos no sistema de saúde, visando responder equitativamente às necessidades das pessoas pela acessibilidade universal através da oferta de medicamentos. Ele ilustra a definição de necessidade comparativa, trazida pelo modelo, que é aquela definida e delimitada a partir do estudo das características da população com o objetivo de lhes prestar serviços de proteção à saúde garantindo a acessibilidade aos medicamentos. Cada sistema de saúde define a cobertura populacional e a fonte de recursos adequada para atingir esse objetivo. Esta definição de necessidade relaciona-se com o princípio da equidade, justiça social, com a preocupação de que a universalidade esteja garantida, até mesmo para os que não sejam portadores de planos de saúde.

$\mathrm{O}$ estudo ${ }^{15}$ informa que, historicamente, a principal forma de obtenção de medicamentos, no Brasil, foi, em seu início, o desembolso direto nas farmácias privadas, modelo mercantilizado e produtor de iniquidades, na medida em que o padrão de acesso e consumo é condicionado pela renda, isto é, na perspectiva do mercado. Entretanto, ao longo da evolução o SUS foi desenvolvendo um modelo de provisão de medicamentos universal e gratuito, por meio dos postos de saúde (PS) e das unidades básicas de saúde (UBS), financiado de forma tripartite, executado e gerido 
no âmbito municipal. A partir de 2004, criou-se o Programa Farmácia Popular do Brasil (PFPB) em paralelo com a distribuição de medicamentos organizada pelo SUS. Em um primeiro momento foi construída uma rede pública própria de farmácias e, a partir de 2006, em sua versão mais abrangente, o Programa passou a credenciar a rede privada de farmácias. Adicionalmente, o PFPB adotou o sistema de copagamento, em que o usuário arca com uma parte do financiamento do medicamento.

Essas transformações no modelo de provisão de medicamentos refletem a influência das distintas hegemonias de política, econômica e ideológica vigentes no período. No período da construção do SUS, a mudança foi impulsionada pela Reforma Sanitária e exigências de melhorias na acessibilidade universal ao sistema de saúde. Este impulso, entretanto, não foi suficiente para evitar o subfinanciamento do SUS, que procurou ser resolvido pela criação do PFPB e que acabou resultando na opção pela parceria com as farmácias privadas como modus operandi de gestão e expansão do PFPB. A parceria com o setor privado, que no início era complementar às farmácias públicas, acabou por se tornar a forma atual do Programa, em simultâneo com a estrutura de provisão de medicamentos do SUS.

Percebe-se aqui que as influências políticas resultaram em uma redefinição do papel do Estado brasileiro na oferta de medicamentos: de um sistema único de distribuição pública e gratuita de medicamentos para um sistema complementar de distribuição pública-privada e com copagamento. Os interesses dos usuários com planos de saúde, que acessam com mais facilidade as consultas médicas, acabaram se fortalecendo frente aos demais, trazendo iniquidades para a acessibilidade aos medicamentos.

De fato, a provisão de medicamentos no SUS é predominante, sobretudo para várias doenças crônicas prevalentes, mas é bastante heterogênea nos vários acessos e conta com problemas financeiros, gerenciais, de infraestrutura e de recursos humanos, além de não ter um sistema de informação unificado implementado, o que dificulta sua monitoração. Já o PFPB atuando por meio das farmácias privadas cadastradas tem um sistema de informação adequado, porém a sua distribuição geográfica é muito menos abrangente, o elenco de medicamentos ofertados mais restrito, e a população que utiliza o Programa é menor do que a que usa a distribuição pela rede pública ${ }^{21}$.

Vale destacar que fica claro que as políticas públicas de assistência farmacêutica sofreram influências históricas, sociais e políticas distin- tas e não foram capazes de reverter totalmente a preponderância do modelo de provisão com desembolso direto pelos consumidores, revelando um desafio importante para garantir a universalidade e corrigir as iniquidades históricas na provisão de medicamentos ${ }^{22}$. Outro aspecto negativo da duplicidade dos sistemas de provisão é que ele acaba interferindo no planejamento da produção da indústria local, pois informa sobre a continuidade das demandas de medicamentos imprecisa ou de modo sobrestimada. De fato, a ausência de informações adequadas e a superposição dos modelos de provisão resultam na incapacidade de o sistema de saúde atingir os seus objetivos de universalidade e gera também iniquidades, na medida em que o PFPB acaba atendendo mais os cidadãos que são proprietários de plano de saúde e moram nas regiões metropolitanas ${ }^{21}$.

A releitura do artigo pelo modelo de Vargas -Peláez et al. ${ }^{1}$ permitiu iluminar os diferentes fatores ideológicos que influenciaram a mudança recente nas políticas públicas brasileiras de provisão de medicamentos e o conflito entre necessidade de saúde e direitos humanos. Se o modelo de provisão do PFPB acabar sendo preponderante em relação ao sistema de distribuição pelos PS e UBS, como apontam as tendências recentes de financiamento, a perspectiva do mercado sobre a da saúde estará em flagrante conflito das necessidades de saúde como direitos humanos.

\section{Demanda de serviços de saúde dependentes de equipamentos}

O terceiro estudo revisitado ${ }^{16}$ ilustra a definição de necessidade expressa pelos serviços de saúde dependentes de equipamentos e suas consequências sobre a acessibilidade desses serviços. O modelo de Vargas-Peláez et al. ${ }^{1}$ destaca a importância dos fatores organização, distribuição geográfica e questões financeiras, e dos fatores facilitadores ao acesso dos usuários, como, por exemplo, status socioeconômico, percepção sobre o sistema e o direito à saúde. Segundo os autores, todos esses fatores influenciam a demanda por serviços de saúde e modulam os desejos de consumo por cuidados.

O estudo ${ }^{16}$ escolheu estudar aspectos da distribuição geográfica e do financiamento e de que modo eles afetam a acessibilidade de serviços médicos de hemodinâmica que são dependentes de equipamentos. Esses serviços médicos de alta complexidade estão diretamente relacionados com a questão da infraestrutura local de equipamentos médicos e hospitalares. 
A organização dos serviços de alta complexidade deveria ser prestados por regiões de saúde, devido ao custo elevado de seu investimento inicial, e a sua distribuição ser feita através das redes de urgência e emergência dada a necessidade da rapidez no cuidado médico relacionado a doenças coronárias. Entretanto, esta regionalização dos serviços de saúde não se deu por igual em todas as regiões do Brasil, gerando algumas preocupações de acessibilidade em relação ao serviço, como é o caso da região que o estudo se debruça que é a região Norte e Noroeste do estado do Rio de Janeiro. Aqui fica evidenciado uma falha do pacto federativo realizado entre os entes federados responsáveis pela organização dos serviços de alta complexidade.

Entre os principais resultados do estudo ${ }^{16}$ fica evidente a existência de poucas equipes médicas especializadas nesse tipo de assistência à saúde e o fato de que esses especialistas são os próprios proprietários dos equipamentos, em sua maioria localizados em hospitais filantrópicos. Não ficou claro qual a quantidade de procedimentos financiados pelo SUS e pelos planos de saúde em cada estabelecimento e sobre os resultados da atenção prestada em termos de tempo de espera e qualidade, aspectos muito relevantes neste tipo de serviço.

Observou-se também que, na região estudada, um dos problemas que dificulta o acesso, apesar da descentralização e regionalização dos serviços, é a falta de regulamentação de uma rede regionalizada para a oferta dos serviços financiados pelo SUS. Em consequência, constatou-se um certo excesso de oferta em um dos municípios, falta em outros, e a necessidade de que esses pacientes circulassem muito para serem atendidos. Outro aspecto observado é que os pacientes com planos de saúde acabavam tendo prioridade no atendimento.

Nessa releitura ficou claro que o arranjo institucional, isto é, um pacto federativo incompleto de descentralização, atende vários interesses constitutivos de setores econômicos que lucram com a atenção à saúde de alto custo e complexidade, e podem estar dificultando o acesso de qualidade e equitativo aos seus usuários. Esse estudo de caso ilustra mais uma vez a dificuldade de se implementar um sistema de saúde universal e equitativo em uma sociedade com grandes problemas de infraestrutura pública de serviços de saúde e desigualdade de renda. Mais do que isso, o arranjo institucional, responsável pela governança da distribuição da demanda de serviços, irá depender da capacidade do Estado de reduzir as desigualdades regionais a partir de uma maior coordenação da reorganização dos interesses constituídos em prol do acesso universal.

\section{Demanda de medicamentos e a influência do mercado}

A motivação da pesquisa realizada nesse último estudo revisitado ${ }^{17}$ foi entender a influência dos fatores de consumo de ansiolíticos em uma comunidade rural, também do norte fluminense. Em particular, detectar os fatores que levam uma população rural, do distrito de Marrecas, da cidade de Campos dos Goytacazes, ao uso excessivo e prolongado de Benzodiazepínicos (BDZs). Este estudo ilustra a definição de necessidade sentida, trazida por Vargas-Peláez et al. ${ }^{1}$ e como ela impacta na acessibilidade do sistema de saúde. Os autores destacam que as necessidades de medicamentos são influenciadas pelo mercado por meio de marcas e difusão junto ao prescritor e ao paciente. $\mathrm{O}$ estudo ${ }^{17}$ problematiza as razões que levam uma população rural ao uso excessivo e prolongado de medicamentos de ansiolíticos em uma comunidade em que os problemas de estresse da vida urbana não estão presentes. Mais do que isso, procura entender porque essa situação persiste mesmo frente à existência de políticas públicas de coibição do uso não racional de medicamentos e de regulação do marketing farmacêutico ${ }^{23,24}$.

O primeiro resultado obtido pelo estudo ${ }^{17}$ constatou uma deficiência dos serviços de saúde oferecidos à população local e um grande número de diagnósticos superestimados e generalizados que priorizam uma terapêutica farmacológica ${ }^{25}$. Também se detectou a falta de orientação médica em relação ao medicamento prescrito e a prática de troca de receitas sem consultas médicas, o que contribui para perpetuar o uso de BDZs nessa população e contraria totalmente o propugnado na política racional de medicamentos. Mostra que apenas a existência dessa política, sem a presença de acompanhamento psicológico e serviço social para o atendimento de pacientes com esses problemas, acaba a inviabilizando.

O segundo resultado diz respeito aos aspectos culturais da população estudada. Observou-se uma supervalorização dos problemas cotidianos e a busca de calmantes como forma de neutralizar de forma imediata sentimentos e emoções tidas como negativas, anormais em uma sociedade midiática. Além disso, pôde-se observar uma distorção do conceito de saúde e a certeza de adquiri-la nas prateleiras e balcões da farmácia. Percebe-se aqui uma clara influência da perspectiva do mercado sobre a perspectiva da saúde na percepção 
da necessidade sentida, fenômeno que a literatura passou a chamar de 'medicalização da vida'17.

Conforme mostram Vargas-Peláez et al. ${ }^{1}$, e que ficou claro com a releitura dos resultados revisitados, as necessidades de saúde não são fatos, mas valores humanos de médicos e pacientes, quer eles percebam isso ou não. Conclui-se que essa compreensão leva, em primeiro lugar, a dificuldade de definir a necessidade de saúde quando ela é fruto de um valor do prescritor médico e do paciente influenciado pelo marketing farmacêutico. Em segundo lugar, a dificuldade de especificação do conceito de saúde que considera os valores da sociedade do conhecimento, em que as técnicas e os procedimentos são mais valorizados do que a saúde do paciente, pela difusão da crença positivista na ciência.

\section{Considerações finais}

A releitura dos estudos na perspectiva das diferentes dimensões das necessidades de saúde e sua influência sobre a acessibilidade do sistema de saúde mostrou que muitas vezes se observa uma sobreposição dos fatores explicativos de cada definição de necessidade de saúde. Por exemplo, o conceito de essencialidade que influencia a definição de necessidade normativa pode ser influenciado pela necessidade sentida, inclusão de medicamentos novos na crença de que o novo é sempre superior ao velho. A principal contribuição do artigo foi ilustrar uma temática muito antiga e ao mesmo tempo atual da economia da saúde que é a problemática definição das necessidades de saúde e sua influência na acessibilidade do sistema de saúde.

A principal lição que se pode extrair é que o equilíbrio entre a perspectiva do mercado e da saúde, representados respectivamente pelo CEIS e pelo SUS, nas diferentes dimensões da definição de necessidades é que contribui para o alcance de um sistema de saúde público e universal. Se por lado, a saúde demanda bens e serviços de altíssima complexidade, alto custo e inovações de fronteira, assim como equipamentos, por outro, a participação do setor saúde contribui com cerca de $9,2 \%$ do produto interno bruto $^{8}$ e poderia ser um setor capaz de dinamizar a economia e elevar o seu padrão de produtividade, alcançando um novo patamar de desenvolvimento com a produção local. Além disso, apostar no desenvolvimento do CEIS seria uma questão muito importante para que a provisão dos bens e serviços de saúde pudesse ser atendida com preços justos, pois se contraporia à concorrência internacional, forçando-a a reduzir os seus preços.

Entretanto, essas atuações pelo lado da oferta parecem não ser suficientes, é preciso, ao mesmo tempo, como a forma de organização da produção de bens e serviços é capitalista, que o Estado tenha um papel não só na coordenação das atividades de produção e na sua regulação, mas também na garantia de oportunidades e distribuição de suas riquezas pelas vias de acessibilidade aos serviços e medicamentos necessários ao atendimento à saúde. A implementação de um sistema de saúde universal e gratuito parece ser a única via para um país, como o Brasil, que ainda sofre de mazelas de desigualdades regionais e concentração de renda.

Enfim, a releitura dos artigos permitiu refletir e iluminar que há muitos desafios pela busca de obtenção dos direitos de saúde numa sociedade capitalista onde a produção e a distribuição dos bens e serviços são organizadas na perspectiva do mercado. Neste sentido, parece imprescindível que o Estado tenha um papel preponderante na busca de uma maior equidade para o alcance dos direitos sociais.

\section{Colaboradores}

L Hasenclever trabalhou na concepção, metodologia, revisão crítica e redação final. CMA Souza trabalhou na concepção e revisão. G Chaves, ALA Peixoto, LV Mattos e JS Viana trabalharam na pesquisa.

\section{Referências}

1. Vargas-Peláez CM, Soares L, Rover MRM, Blatt CR, Mantel-Teeuwisse A, Buenaventura FAR, Restrepo LG, Latorre MC, López JJ, Bürgin MT, Silva C, Leite SN, Rocha FM. Towards a theoretical model on medicines as a health need. Soc Sci Med 2017; 178:167-174.

2. Soares L. O acesso ao serviço de dispensação e a medicamentos: modelo teórico e elementos empíricos [tese]. Florianópolis: Universidade Federal de Santa Catarina; 2013. 
3. Gadelha C, Barbosa P, Maldonado J, Vargas M, Costa L. O Complexo Econômico-Industrial da Saúde (CEIS): conceitos e características gerais. VPPIS/FIOCRUZ; Informe CEIS 2010; 1(1):2-16.

4. Ouverney AM, Noronha JC. Modelos de organização e gestão da atenção à saúde: redes locais, regionais e nacionais. In: Fundação Oswaldo Cruz. A saúde no Brasil em 2030 - prospecção estratégica do sistema de saúde brasileiro: organização e gestão do sistema de saúde. Rio de Janeiro: Fiocruz/Ipea/Ministério da Saúde/Secretaria de Assuntos Estratégicos da Presidência da República. Vol. 3. Rio de Janeiro: Scielo Books; 2013. p. 143-82.

5. Hasenclever L, Paranhos J, Chaves GC, Damasceno C. Uma análise das políticas industriais e tecnológicas entre 2003-2014 e suas implicações para o Complexo Industrial da Saúde. In: Hasenclever L, Oliveira MA, Paranhos J, Chaves GC, organizadores. Desafios de operação e desenvolvimento do Complexo Industrial da Saúde. Rio de Janeiro: E-papers Serviços Editoriais Ltda; 2016. p. 99-126.

6. Bahia L. A saúde nos governos petistas: políticas públicas antagônicas. In: Maringoni G, Medeiros J, organizadores. Cinco mil dias: o Brasil na era do lulismo. São Paulo: Boitempo/Fundação Lauro Campos; 2017. p. 223-8.

7. Hasenclever L, Paranhos J, Chaves GC, Oliveira MA, organizadores. Vulnerabilidades do Complexo Industrial da Saúde - Reflexos das políticas industrial e tecnológica na produção local e assistência farmacêutica. Rio de Janeiro: E-papers Serviços Editoriais Ltda; 2018.

8. Instituto Brasileiro de Geografia e Estatística (IBGE). Conta-Satélite de Saúde, Brasil: 2010-2017. no 71. Rio de Janeiro: IBGE; 2019.

9. Bradshaw J. The taxonomy of social need. In: McLachlan G, editor. Problems and Progress in Medical Care: essays on current research. 7th series. London: Oxford University Press; 1972. p. 71-82.

10. Willard LD. Needs and medicine. J Med Philos 1982; 7(3): 259e274.

11. Max-Neef M, Elizalde A, Hopenhayn M. Desarrollo a escala Humana: conceptos, aplicaciones y algunas reflexiones. $2^{a}$ ed. Montevideo: Editorial Nordan- Comunidad;1998.

12. Sen A. Desenvolvimento como Liberdade. São Paulo: Cia. das Letras; 1999.

13. Hasenclever L, Paranhos J, Chaves GC, Damasceno C. Uma análise das políticas industriais e tecnológicas entre 2003-2014 e suas implicações para o Complexo Industrial da Saúde. In: Hasenclever L, Oliveira MA, Paranhos J, Chaves GC, organizadoras. Desafios de operação e Desenvolvimento do Complexo Industrial da Saúde. Rio de Janeiro: E-papers; 2016. p. 99-126.

14. Chaves GC, Oliveira MA. Estratégias governamentais para redução de preços de medicamentos em situação de monopólio: produção local e enfrentamento da barreira patentária. In: Hasenclever L, Paranhos J, Chaves GC, Oliveira, MA, organizadoras. Vulnerabilidades do Complexo Industrial da Saúde - Reflexos das políticas industrial e tecnológica na produção local e assistência farmacêutica. Rio de Janeiro: E-papers; 2018. p. 181-212.
15. Luiza VL, Silva RM, Mattos LV, Ligia B. Fortalezas e desafios dos modelos de provisão e financiamento de medicamentos. In: Hasenclever L, Paranhos J, Chaves GC, Oliveira MA, organizadoras. Vulnerabilidades do Complexo Industrial da Saúde - Reflexos das políticas industrial e tecnológica na produção local e assistência farmacêutica. Rio de Janeiro: E-Papers; 2018. p. 213252.

16. Andreazzi MFS, Hasenclever L, Peixoto ALA. Arranjos institucionais em serviços dependentes de equipamentos para diagnóstico e terapêutica de doenças cardiovasculares: um estudo sobre as regiões Norte e Noroeste do estado do Rio de Janeiro. In: Hasenclever L, Paranhos J, Chaves GC, Oliveira MA, organizadoras. Vulnerabilidades do Complexo Industrial da Saúde - Reflexos das políticas industrial e tecnológica na produção local e assistência farmacêutica. Rio de Janeiro: E-papers; 2018. p. 253-276.

17. Viana JS. Política de uso racional de medicamentos: $o$ consumo de ansiolíticos na localidade rural de Marrecas, Campos dos Goytacazes - RJ [dissertação]. Campos dos Goytacazes: Universidade Cândido Mendes; 2018.

18. Hasenclever L, Fialho BC, Klein HE, Zaire C. Economia industrial de empresas farmacêuticas. Rio de Janeiro: E-papers Serviços Editoriais; 2010.

19. Hasenclever L, Paranhos J, Klein HE, Coriat B. Reflexos das políticas industriais e tecnológicas de saúde brasileiras na produção e fornecimento de ARVs genéricos pos-2005. In: Possas C, Larouze B, organizadores. Propriedade Intelectual e Políticas Públicas para o Acesso aos Antirretrovirais nos Países do Sul. Rio de Janeiro: E-papers; 2013. p. 127-142.

20. Chaves GC, Azeredo TB, Vasconcelos DMM, Mendonça-Ruiz A, Hasenclever L, organizadores. Produção Pública de Medicamentos no Brasil: capacitação tecnológica e acesso a medicamentos. Rio de Janeiro: E-papers; 2018.

21. Silva JEM. Arranjos de provisão de medicamentos básicos e essenciais: análise do Programa Farmácia Popular do Brasil [tese]. Campos dos Goytacazes: Universidade Cândido Mendes; 2019.

22. Bahia L. Trinta anos de sistema único de saúde pública (SUS): uma transição necessária, mas insuficiente. Cad Saude Publica 2018; 34(7):e00067218.

23. Paumgartten FJR, Nascimento AC. Regulação Sanitária de Medicamentos. In: Osório-de-Castro CGS, Luiza VL, Castilho SR, Oliveira MA, Jaramillo NM, organizadores. Assistência Farmacêutica. Gestão e prática para profissionais de saúde. Rio de Janeiro: Fiocruz; 2014. p. 207-220.

24. Esher A, Coutinho T. Uso racional de medicamentos, farmaceuticalização e usos do metilfenidato. Cien Saude Colet 2017; 22(8):2571-2580.

25. Pombo MF. Medicalização do sofrimento na cultura terapêutica: vulnerabilidade e normalidade inalcançável. Rev Electron Comun Inf Inov Saude 2017; 11(1):114.

Artigo apresentado em 22/04/2020

Aprovado em 11/02/2021

Versão final apresentada em 13/02/2021

Editores-chefes: Romeu Gomes, Antônio Augusto Moura da Silva 